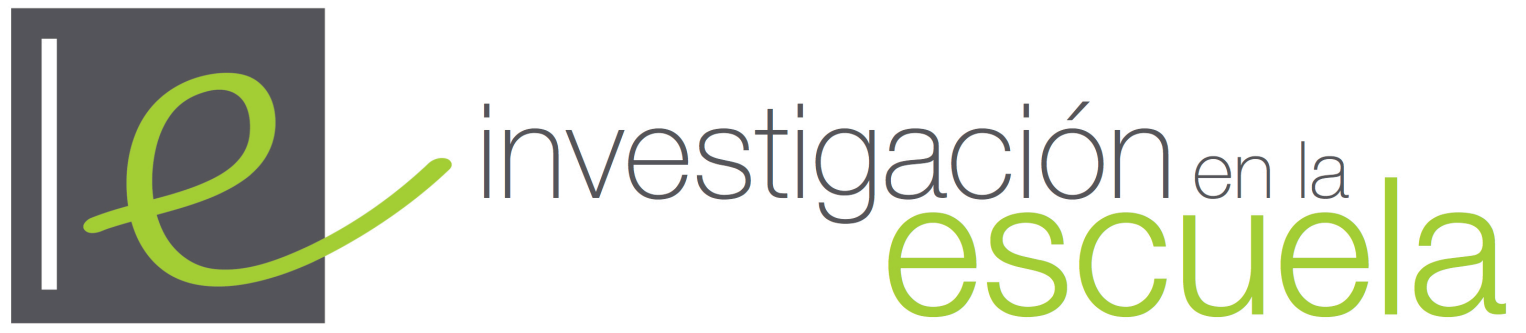

Revista internacional de investigación e innovación educativa

\title{
La acción educativa en el aula desde planteamientos innovadores: un estudio intensivo
}

\author{
Virginia Morcillo Loro \\ Universidad de Huelva \\ España
}

Citación: Morcillo Loro, V. (2017). La acción educativa en el aula desde planteamientos innovadores: un estudio intensivo. Investigación en la Escuela, 92, 1-18. Recuperado de: http://www.investigacionenlaescuela.es/articulos/R92/R92-1

Resumen: Se presenta un estudio intensivo compuesto por el desarrollo de dos estudios de casos únicos que desenvuelven su actividad didáctica a partir de procesos de aprendizaje por investigación. Específicamente, se depara en el papel que juegan las variables (materiales curriculares, participación, gestión del espacio y del tiempo...) que inciden en el aula, a la hora de definir la práctica docente. Participan dos maestras de educación infantil y primaria respectivamente, que han cooperado voluntariamente. Su representatividad viene avalada por la duración de la experiencia -un año-, el interés expresado y su relación con contextos diferentes, todo lo cual permite comparaciones relevantes. La recogida de datos ha sido a partir de instrumentos variados (entrevistas, diarios, observaciones, entre otros). Para organizar la información contamos con una tabla que agrupa las evidencias de modo accesible. En concreto se expone cómo aunque ambas se inclinan hacia el aprendizaje por investigación, las diferencias entre ellas son notables.

Palabras clave: "Acción educativa"; "estudio intensivo"; "educación infantil”; "educación primaria"; "estudio de casos". 
Educational action in the classroom from innovative approaches: an intensive study

Abstract: We present an Intensive Study composed by the development of two unique case studies that develop their didactic activity from research learning processes. Specifically, it lies in the role of the variables that affect the classroom, when defining the teaching practice. There are two primary and elementary education teachers, who cooperate voluntarily. Its representativeness is supported by the duration of the experience, the expressed interest and its relation with different contexts, all of which allows relevant comparisons. The data collection has been from various instruments. To organize the information we have a table that groups the evidence in an accessible way. In particular, it is exposed how, although both are inclined towards learning by investigation, the differences, among them, are remarkable.

Key words: "Educational action"; "intensive study"; "early childhood education"; "primary education"; "study of cases".

\section{Action éducative dans la classe des approches novatrices: une étude intensive}

Resumè: Une étude intensive comprend l'élaboration de deux études de cas individuels qui développent leurs activités d'enseignement des processus de recherche est présenté apprentissage. Plus précisément, il tient dans le rôle des variables qui affectent la classe lors de la définition pratique éducative. Impliquer deux enseignants de la maternelle et les écoles primaires, qui ont volontairement coopératif. Représentativité est garantie pour la durée de l'expérience, exprimé leur intérêt et leur relation avec les différents contextes, qui permet des comparaisons pertinentes. La collecte des données a été de divers instruments. Pour organiser les informations que nous avons une table que les groupes de la preuve de manière accessible. Plus précisément, il explique comment bien que les deux penchent vers la recherche d'apprentissage les différences entre eux sont remarquables.

Mostsclé: "Action éducative"; "étude intensive"; "éducation infantile"; "éducation primaire"; "étude de cas".

\section{Introducción}

Cuando a día de hoy seguimos planteándonos si es posible y necesaria otra escuela (Carnie, 2003; Pozuelos, Romero, García y Morcillo, 2010), mostramos cierta pesadumbre si deparamos en el horizonte didáctico-educativo más extendido. Anhelamos otra escuela pero seguimos pensando que la educación está aún a medio camino, que se transita hacia otras propuestas pero no acaban de llegar. Vivimos en una sociedad que se tambalea entre la incertidumbre y el titubeo -realidad además presente en el terreno escolar-. Una sociedad sumida en constantes cambios, a un ritmo hiperacelerado y bajo una oleada de transformaciones en el conglomerado social, que afecta lógicamente al campo educativo de manera directa.

Está claro que el horizonte escolar mundial está cambiando y lo está haciendo avivadamente. Esta realidad con la que nos encontramos busca las mejores respuestas para una infancia (Giroux, 2003; Pérez, 2004) que así lo reclama y demanda. Sírvase de ejemplo el discurso de Alison Cook-Sather (2007), del cual tomamos como reseña una de sus premisas más imperativas, autorizar al alumnado como agente de cambio y transformación en educación. Autorizar a los niños y a las niñas significa asegurar que existen espacios dialógicos y deliberativos en los que se escuchen y legitimen sus derechos y perspectivas, se tengan en cuenta y, al mismo tiempo, que sean incorporados a los intentos de reforma: escuchar de verdad implica dar respuestas. 
Dentro de la Agenda de Lisboa, la Unión Europea se ha propuesto (ahora renovada en los nuevos objetivos de referencia para 2020) el éxito educativo para todos. En este sentido, la flecha apunta hacia la dinamización de los centros escolares de modo que puedan conseguir buenos aprendizajes para todos los alumnos; más allá del voluntarismo y confianza en su profesorado (Bolívar, 2012). Desde esta consideración,

se subraya la idea de pasar de una política, [...] basada en lógicas de arriba-abajo, de matriz jerárquica y burocrática, a formas de gobierno en red o de gobernanza. Desde esa perspectiva, se trata de saber responder a los nuevos retos, incorporando en los procesos de decisionales a los actores, colectivos y personas involucradas en los mismos (Subirats, 2010, p. 33).

Esta apreciación denota el desencanto sobre la posibilidad que las reformas externas han demostrado para transformar la realidad educativa en el último tercio del siglo pasado. Tal desaliento ha conducido a tomar la escuela como un lugar estratégico para el cambio y la mejora generada desde abajo.

Así lo señala Thurler (2004) cuando rotula que el aula juega necesariamente un papel relevante en la construcción del sentido del cambio (Fullan, 2002) porque constituye el entorno del trabajo diario, la comunidad de pertenencia y el contexto de la educación. Escudero (1999, p. 272) apuesta, también, por la "recuperación del aula y los procesos de enseñanza-aprendizaje". Por tanto, el aula es el punto de mira de la institución educativa y bajo este prisma reconstruir su imagen.

Este nuevo perfil iconográfico considera la particularidad de cada escolar desde su desarrollo integral, una filosofía amigable con la inclusión escolar (Giné, Durán, Font y Miquel, 2009), la justicia social (Belavi y Murillo, 2016), la educación para la ciudadanía (Argos, 2008), el Movimiento por los Derechos de la Infancia (Declaración de los Derechos del Niño, 1989) y el Movimiento de Mejora Escolar (Murillo y Krichesky, 2015). Asimismo, toma como eje la voz del alumnado para la mejora escolar (Fielding, 2011; Susinos y Ceballos, 2012). En el nuevo imaginario social, los niños y las niñas como ciudadanos, de una sociedad democrática y plural, han de participar libre y voluntariamente acorde al desarrollo de sus capacidades, motivaciones, intereses y potencialidades. Los niños y las niñas de hoy y del mañana, reclaman participar en los asuntos públicos, ofreciendo su visión particular, desde una perspectiva más libre, imaginativa, realista, creativa, original, más justa, equitativa, sincera, limpia y menos sesgada que la de los adultos. Por otra parte, el aula se hace eco de la gramática tecnológica en el proceso de enseñanza-aprendizaje, otorgando un carácter relevante al papel del estudiante y del docente, la revalorización del trabajo colaborativo entre profesionales desde la perspectiva del liderazgo compartido o distribuido (Harris y Jones, 2010), la incorporación de nuevas competencias clave y saberes fundamentales para una educación del siglo XXI desde una visión integrada del conocimiento (Gimeno, 2005), la participación de la comunidad educativa en proyectos educativos (Parrilla, Muñoz y Sierra, 2013) y la reflexión de la práctica docente (Trillo Alonso, 1994).

$\mathrm{Al}$ mismo tiempo, la reconstrucción de la acción educativa nos dirige hacia el replanteamiento de mejoras en la organización del tiempo, en la organización espacial y en el empleo, funcionalidad y utilidad de los recursos materiales, en el currículum y la dinámica de enseñanza.

Somos conscientes que el aula puede llegar a albergar algunas soledades, incluso puede resultar un espacio complejo y difícil. En algunos casos, el aula supone ese lugar o espacio en el que la jerarquización se integra como una epidemia casi irremediable -hablamos del papel del docente sobre el papel del alumno-. En otros, sin embargo, la horizontalidad apremia el trabajo conjunto que docentes y alumnos realizan a diario. Es, por tanto que concebimos el aula como: 
Un entorno complejo, avivado, cambiante, que acciona el motor de arranque del proceso de enseñanza-aprendizaje. Un espacio singular en otros sub-escenarios específicos, con cualidades comunes, así como diferentes. Un lugar motivado y que motiva, que debería incitar al logro de aprendizajes significativos y relevantes, en un espacio en el que todo el alumnado tiene cabida. Un contexto, un microsistema, en el que el diálogo, la democracia, las interacciones, constituyen y definen la vida misma o la esencia de ese espacio. Un lugar donde la afectividad, la risa, el juego y la investigación se consideran como los pilares que sostienen la misma. En definitiva, el aula como el oxígeno que nos mantiene vivos, como el agua que nos mantiene hidratados, como la tierra por donde pasamos pero que, según la visión que tengamos de ella, se puede convertir en un espacio para la soledad o en el lugar que hace avanzar y evolucionar al alumnado y al profesorado.

\section{Encuadre metodológico}

El corazón de nuestra investigación palpita con la necesidad de encontrar estímulos y mecanismos que nos permitan conocerlo y describirlo para seguir su propio biorritmo. El estudio que aquí presentamos es parte de uno de los tres sub-estudios de una investigación más amplia. Se denomina "Estudio Intensivo" y su razón de ser radica en comprender una determinada realidad social, cultural y educativa. La denominación de "Intensivo" viene marcada por la necesidad de combinar instrumentos y estrategias de naturaleza abierta y flexible para describir y comprender la realidad objeto de estudio.

Se presentan dos estudios de caso únicos, los cuales se muestran como un procedimiento para explorar, conocer y comprender las múltiples variables que intervienen en la dinámica de aula, con la finalidad de analizar cómo funciona, desde planteamientos innovadores reconocidos y asentados.

Para la selección de los casos aplicamos lo que Goetz y LeCompte (1998, p. 98) definen como selección basada en "criterios simples". En líneas generales, para este estudio se han seleccionado los siguientes criterios que justifican la elección de ambos casos: colaboración voluntaria, ubicación: centros educativos públicos, participación abierta, prácticas reconocidas, fases de desarrollo profesional diferente, y publicaciones sobre experiencia profesional y práctica educativa -no es un requisito imprescindible pero sí considerable-.

Con el fin de recabar información y comprender las líneas de cada experiencia, se han empleado una serie de técnicas e instrumentos de corte cualitativo que estructuramos de la siguiente forma (Gutiérrez, 2006): a) instrumentos para observar la realidad (diario docente y memorándum, observaciones de aula, conversaciones informales, fotografías y vídeos, documentos del centro) y b) instrumentos para interrogar la realidad (entrevistas personales -docentes-, entrevista biográfico-narrativa y entrevistas colectivas -alumnado y familias-, triangulación).

Los principios éticos que estuvieron presentes para el desarrollo de este estudio fueron: a) la negociación, b) la colaboración, c) la confidencialidad, d) la imparcialidad, e) la equidad y f) el compromiso con el conocimiento.

\section{Dos casos únicos con tintes innovadores, bajo un mismo paraguas geográfico}

La realidad contextual de ambos casos es la misma, en cuanto a su ubicación. La localidad de Punta Umbría (Huelva), alberga diferentes realidades bajo un mismo espacio diverso en relación al nivel social, económico y cultural del municipio. Dos centros cuya idiosincrasia queda 
determinada por una localización geográfica específica. Hablamos de dos zonas con niveles socioeconómicos y culturales disímiles. No obstante, ambas realidades se ven influenciadas por un entorno natural y social con cualidades genuinas.

Ambos casos se encuentran enlazados por una línea imaginaria desde hace décadas. Un centro educativo con raíces profundas en los Movimientos de Renovación Pedagógica e Innovación Educativa. Un espacio donde se codeaban y formaban docentes con una visión alternativa a la enseñanza convencional. La escuela no es lo que era. La excesiva burocratización de las instituciones escolares junto al individualismo en el colectivo docente, han perfilado un retrato diferente de la misma. El ajuar de la escuela ha cambiado, la cultura escolar presenta matices diferentes. Pero, aun, sigue albergando a docentes que ofrecen tentativas hacia esos principios pedagógicos y filosóficos que emanaba en sus primeros años de creación.

Julia y Carmen, dos retratos singulares, con trayectorias profesionales diferentes. En sus primeros años de docencia, Julia asistía a seminarios de enseñanza alternativa en el CEIP Los Pinos, donde en la actualidad, ejerce docencia Carmen.

Dos profesionales con un planteamiento común: la mejora del campo educativo desde la participación de sus protagonistas, los escolares.

Tabla 1

Rasgos propios de cada caso

\begin{tabular}{|c|c|c|c|c|c|c|}
\hline $\begin{array}{l}\text { Nombre } \\
\text { docente }\end{array}$ & $\begin{array}{c}\text { Estudio de } \\
\text { caso }\end{array}$ & $\begin{array}{c}\text { Centro } \\
\text { educativo }\end{array}$ & $\begin{array}{c}\text { Etapa } \\
\text { educativa }\end{array}$ & $\begin{array}{l}\text { Ubicación } \\
\text { geográfica }\end{array}$ & $\begin{array}{c}\text { Contexto } \\
\text { Socioeconómico }\end{array}$ & Características \\
\hline Julia & 1 & $\begin{array}{c}\text { CEIP } \\
\text { Virgen del } \\
\text { Mar }\end{array}$ & Infantil & $\begin{array}{c}\text { Punta } \\
\text { Umbría } \\
\text { (Huelva) }\end{array}$ & Medio & $\begin{array}{c}\text { El aula como } \\
\text { espacio } \\
\text { innovador, } \\
\text { creativo y } \\
\text { experimental } \\
\text { Proyectos de } \\
\text { trabajo }\end{array}$ \\
\hline Carmen & 2 & $\begin{array}{l}\text { CEIP Los } \\
\text { Pinos }\end{array}$ & Primaria & $\begin{array}{c}\text { Punta } \\
\text { Umbría } \\
\text { (Huelva) }\end{array}$ & Medio-bajo & $\begin{array}{c}\text { Comunicación } \\
\text { y expresión en } \\
\text { el aula } \\
\text { Tareas } \\
\text { integradas }\end{array}$ \\
\hline
\end{tabular}

Caso 1: el aula, un espacio innovador, creativo y experimental. Trabajando con proyecto de trabajo en la etapa de infantil

La docente elegida es Julia. Lleva en el cuerpo docente desde el año 1988, año en el que aprobó las oposiciones. Cuenta con más de 25 años de práctica y está entre el colectivo docente de un centro público de infantil y primaria, en una de las escuelas consideradas de toda la vida desde el año 1998.

El edificio escolar es bastante antiguo, finales de los sesenta, pero con una remodelación en el 2003, por lo que las dependencias interiores están adaptadas a las nuevas necesidades y demandas y su aspecto exterior resulta suficientemente conservado y actual.

El centro es de dos líneas completas desde infantil a primaria. Desde hace cuatro cursos, está catalogado de "Educación Compensatoria". El profesorado adscrito es al completo con destino definitivo, con un porcentaje elevado de maestras. 
El equipo de la etapa de Educación Infantil está formado por siete maestras (dos por nivel más una de apoyo) y dos monitoras que ayudan en tareas asistenciales a los niveles de tres años.

El estudio de caso se encuadra principalmente en el proyecto de trabajo "Estar en forma es genial". Un proyecto de investigación escolar enmarcado en el ámbito de la "Alimentación Humana" del Proyecto Curricular Investigando Nuestro Mundo INM (6-12) (Pozuelos, González y Travé, 2008).

Cuando hablamos del aula discutimos de la esencia del proceso de enseñanza-aprendizaje. El aula de Julia es especial y, lo es, por varios motivos: por la docente que guía y orienta el proceso educativo, por los protagonistas que dan coherencia y sentido al aula y, por los agentes que participan, así como por otra serie de variables que la condicionan e influencian. En su aula se tejen palabras como motivación, creatividad, cooperación, experimentación y participación. Son principios que atestiguan y dan credibilidad a un contexto enmarcado por enfoques innovadores y alternativos.

Su aula tiene vida, es dinámica y envolvente. Se caracteriza en función de los proyectos que se desarrollan y se completa con las experiencias de sus escolares. Es un espacio físico, pero temporalmente, porque se abre y extiende hacia el entorno. El contacto con la realidad sumerge al aula en un espacio donde se potencia la curiosidad y la observación, la imaginación y la indagación.

El aula permite la incorporación de proyectos que suponen una aventura en la que debatir, dialogar, descubrir, comunicar...

Es un aula interactiva, diversa, social, personal y académica; donde los niños y las niñas tienen la oportunidad de aprender a ser, a vivir y convivir. Un álbum de imágenes y metáforas que evolucionan a ritmo del tiempo.

La dinámica de enseñanza se fundamenta en los proyectos de trabajo (Pozuelos, 2007). El currículum en la acción se inicia con hipótesis de trabajo ("interrogantes del alumnado"). Su preocupación más inmediata es que el alumnado viva la escuela, participe y tenga curiosidad por aprender.

Para el tratamiento del currículum recurre desde el curso 2002 a proyectos de trabajo e investigación escolar. Un enfoque que surgió a raíz de la colaboración con un grupo de investigación de la Universidad de Huelva. Su inicio fue como una auténtica "revolución" de la práctica docente, actuando como referente para asentar las bases de su labor como profesional.

Julia huye de convencionalismos y apuesta por un aula en la que el alumnado tenga la oportunidad de disfrutar de su aprendizaje. Para ello, incorpora temáticas que despierten interés y motivación en los escolares, con la finalidad de que hagan suyo el proceso educativo. Sus dudas e interrogantes fluyen a diario en el aula.

Para la selección de temáticas tiene en cuenta que sean motivadoras para los niños y niñas, que se adecúen a los contenidos y las competencias que aparecen recogidos en el currículum y que tengan relevancia social.

La secuenciación del proyecto se dispuso tal y como se recoge en la figura 1. 


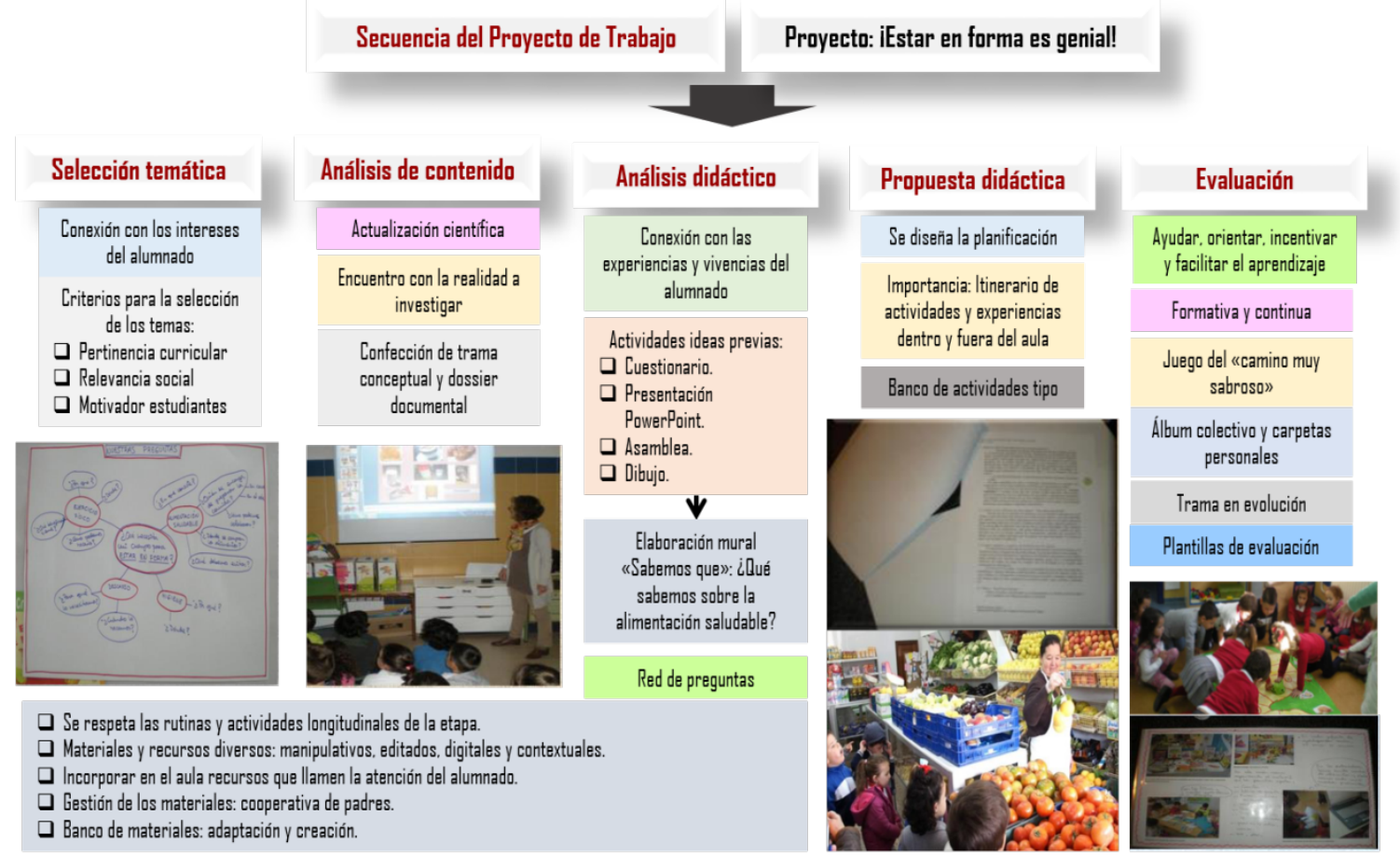

Figura 1. Secuenciación del desarrollo del proyecto "Estar en forma es genial"

El aula se dibuja con la historia del curso. Contempla el espacio físico desde la perspectiva del alumnado, sus producciones son las que dan vida al espacio. El aula se contextualiza con las producciones de los escolares. Se transforma según los intereses de los niños que, poco a poco, la van a ir conquistando y haciendo suya. Julia organiza el espacio pensando en los niños y niñas, es estimulante, accesible, flexible, funcional, estética y agradable a los sentidos.

Se encuentra distribuida en rincones y talleres (ordenador y experimentos), con espacios para actividades individuales (juego libre), en pequeños grupos (mesas de trabajo) y en gran grupo (zona de asamblea). Consta básicamente de áreas especializadas (rincón de la casita, rincón de la comida, rincón de la biblioteca, rincón-mural...) y las zonas multifuncionales (áreas y mesas de trabajo).

La asamblea constituye el escenario privilegiado para que los escolares puedan llevar su mundo al aula. Los materiales se encuentran al alcance del alumnado, son diversos y los espacios se encuentran adaptados a las características del alumnado.

El espacio del aula se define por: i. Espacios para pensar, dialogar, comunicar y expresar, ii. Espacios para la creación, el trabajo en equipo, la definición de roles y la ayuda mutua, iii. Espacios que evolucionan con cada proyecto, iv. Espacios para observar cambios, realizar experimentaciones, v. Espacios para reciclar, vi. Espacios para la organización de materiales, vii. Espacios para cobijar el trabajo individual del alumnado, etc.

Con respecto a la dimensión temporal, los tiempos se desenvuelven con naturalidad, sin una plantilla rígida. La organización temporal y espacial es coherente entre sí. Cada día de clase se estructura en función de tiempos. El tiempo de las actividades se encuentra ligado al espacio en el que se realiza cada una de ellas: hay un tiempo para debatir, dialogar, recitar, cantar... en la asamblea, otro tiempo para jugar en los rincones, un tiempo para trabajar en los talleres, así como 
el tiempo del recreo, el tiempo de la merienda y el tiempo para las actividades planificadas y dirigidas.

Gracias a las actividades que Julia diseña e implementa, potencia la cohesión y el consenso entre el propio alumnado, así como también entre ella y su compañera de nivel. Propicia ciertas rutinas y hábitos que hacen más ágiles las relaciones, establece normas claras y precisas sobre las relaciones y acciones de los escolares y de ella como docente y elige el agrupamiento adecuado, ofreciendo la oportunidad a los escolares para que decidan cómo quieren agruparse.

Gestiona la convivencia constituyendo una verdadera comunidad de aprendizaje, en las que las relaciones se hacen extensibles a otros agentes que participan de la vida del aula. Facilita el conocimiento de todos los protagonistas del aula y el acercamiento de unos hacia otros. Además, las relaciones no solamente se producen en el interior de la clase sino que se amplían a otros contextos.

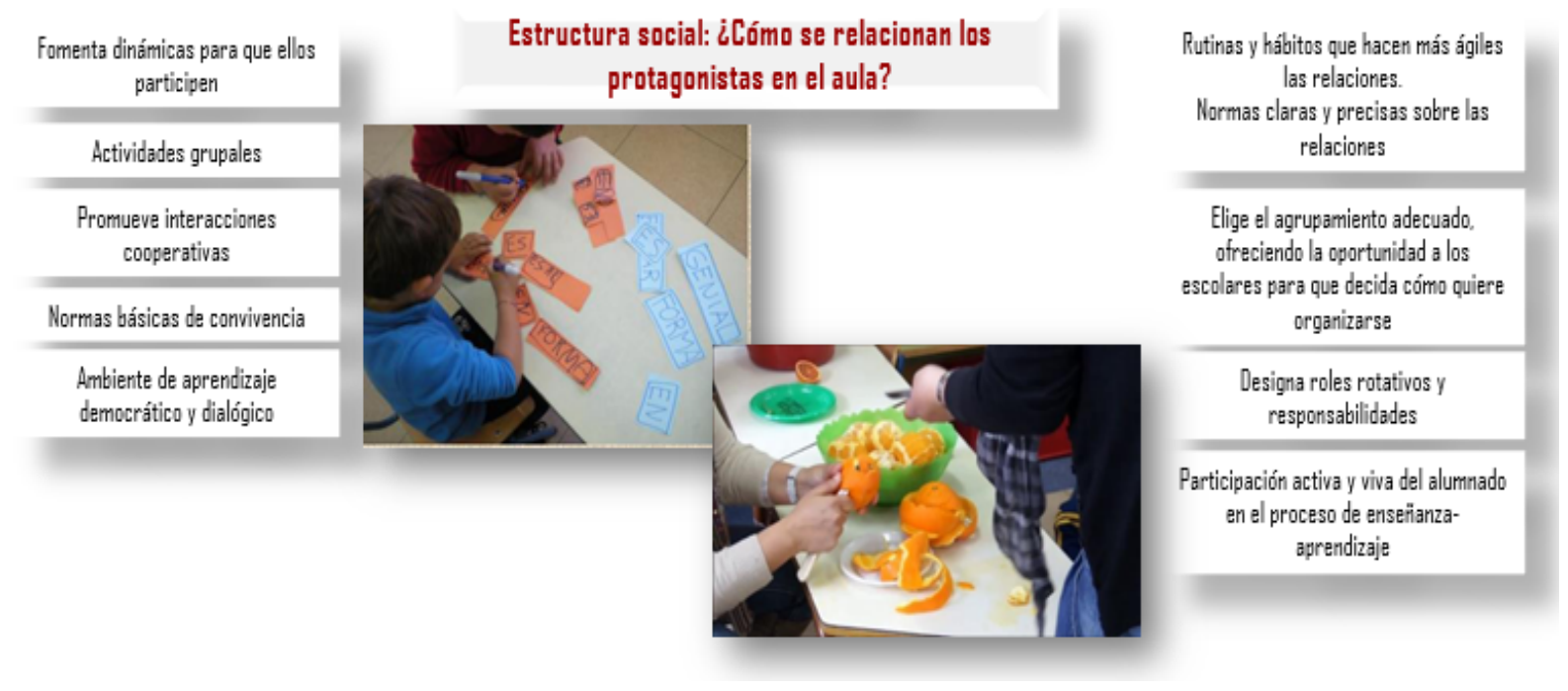

Figura 2. Estructura social del aula

La participación comunitaria prima en las experiencias educativas innovadoras. Las puertas se abren para dar paso a una red de personas que constituyen verdaderos referentes para el aprendizaje del alumnado. Es una polifonía de voces de las que aprender y que, a su vez, sirven de apoyo al proceso de enseñanza-aprendizaje. El aula de Julia se caracteriza por un devenir de personas que "facilitan" la acción educativa. Para ella, contar con la participación y la colaboración familiar es uno de los puntos que refuerza el trabajo por proyectos. La responsabilidad del aprendizaje del alumnado no recae únicamente en el profesorado.

Finalmente, en la figura 3 exponemos los obstáculos y los elementos que favorecen la actividad en el aula. 


Obstáculos y necesidades
$\square$ Individualismo y presión de una evaluación.
$\square$ Considera que la Administración actúa con la
razón y no desde el corazón.
$\square \quad$ Antes: existía una comunicación diferente e
ilusión por hacer.
$\square$ No existe conciencia real entre el
profesorado para reflexionar y ser críticos
con la labor profesional.
Actitud pesimista en el cambio.
Falta de tiempo.
Necesidad de contar con grupos de trabajo y
con asesoramiento externo.
Fallo en recursos tecnológicos.

Figura 3. Obstáculos y elementos que favorecen la actividad en el aula

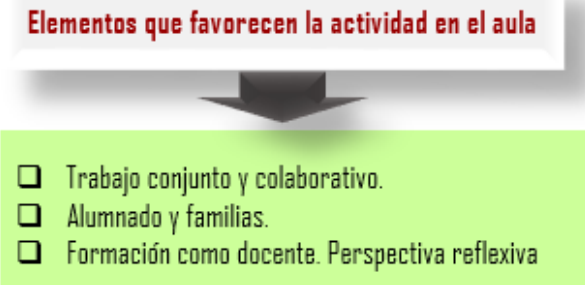

"Con toda la formación he experimentado cambios muy notables, mi práctica es producto de todo lo que he ido aprendiendo a lo largo de los años"(EBDD). "Cuanto más formación tienes más capacidad y más facilidad para seguir" avamzando"(EBDD)

"Con este provecto he querido que mis escolares conozcan y adquieran hábitos saludables de modo funcional. Para este propósito y contando con sus preguntas he desarrollado una experiencia de aprendizaje por investigación (proyecto de trabajo).Lógicamente, este proceso nos ha llevado a realizar actividades y experiencias de muy distinta naturaleza: averiguar, discutir. comprobar, elaborar, visitar y sacar conclusiones... todo ello con la intención de construir un conocimiento cada vez más elaborado. No se trata de saber para la escuela, buscamos conocer desde la vida y para todos los contextos $\gamma$ eso ocurre cuando pasamos por distintas situaciones y múltiples acciones. Solo desde el interés se despierta el entusiasmo y esta es la prueba" (DDM)

\section{Caso 2: la comunicación y la expresión en el aula de la mano de sus protagonistas. Ejes de una dinámica innovadora}

El centro educativo data del año 1987. El edificio escolar es antiguo, pero para acondicionar el mismo a las nuevas necesidades y demandas, se ha remodelado o adaptado algunas de sus dependencias y su fachada resulta suficientemente conservada y actual. En cuanto a su ubicación, señalar que se encuentra situado en una de las salidas principales de Punta Umbría, por lo que su acceso es cómodo y de fácil localización.

Actualmente la realidad socio-económica del centro ha dado un giro. En voz de Carmen, hay casos de absentismo localizados en la escuela, entre otras situaciones más complejas. El centro se hace eco de esta realidad, asumiendo el papel de "Escuela Espacio de Paz", así como otros proyectos.

Carmen es una maestra joven, con escasos años de docencia. Unos referentes familiares ligados a la música con tintes clásicos. Es una docente preocupada por el futuro de sus estudiantes, por su formación. Entre sus principios pedagógicos destacan la participación activa del alumnado, la importancia de la comunicación oral y escrita, la conexión con el entorno acercando al alumnado a problemáticas sociales, la investigación y la indagación, unido a la aplicación de las tecnologías de la comunicación y de la información en el aula.

El concepto de aula se encuentra ligado al grupo humano que engloba. Es un aula abierta a nuevos ambientes de aprendizaje donde las tecnologías educativas tienen cabida. El uso de las TIC se emplea para la generación de conocimiento compartido y para el desarrollo del trabajo colaborativo. Los escolares se involucran en actividades de aprendizaje dinámicas.

Un aula que se encuentra iniciándose en los proyectos de investigación escolar desde las tareas integradas, donde el rol de Carmen es de guía y orientador, ofreciendo pistas al alumnado 
para que construyan su aprendizaje. Potencia la educación emocional, el pensamiento crítico y las responsabilidades individuales. No existen barreras para la "palabra", ni silencios que encarcelen una comunicación fluida y democrática. Se respetan los Derechos de la Infancia y se acerca a los estudiantes al ámbito cultural, musical y formativo.

Las tareas integradas se constituyen como eje vertebrador de la dinámica de enseñanza. Con el "Proyecto Europa" comprobamos como el currículum se estructura en función de tareas integradas.

Carmen hace uso de las tareas integradas para la resolución de problemas. Se trata de un conjunto de actividades debidamente organizadas y enlazadas entre sí para conseguir un fin determinado, con lo que se persigue: i. El conocimiento en acción. La creación de un producto social relevante y significativo, ii. El alumnado adquiere habilidades, aptitudes, actitudes y iii. Un aprendizaje útil para la vida real.

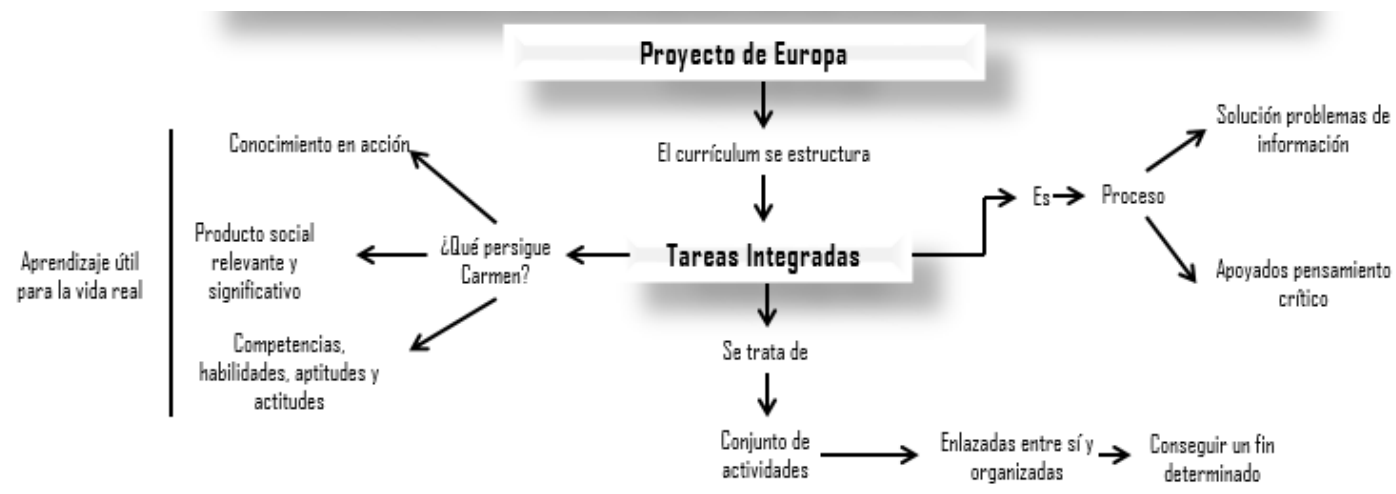

Figura 4. Concepto de tarea integrada para la docente

Una tarea implica un proceso que consta de diferentes partes diferenciadas: un inicio, un conjunto de acciones intermedias y un fin. Para ello, Carmen selecciona las tareas que son más necesarias en la vida real, por ejemplo, aquellas que potencian la comunicación oral. Son proyectos integrados del área de Conocimiento del Medio donde se trabajan diversos enfoques. 


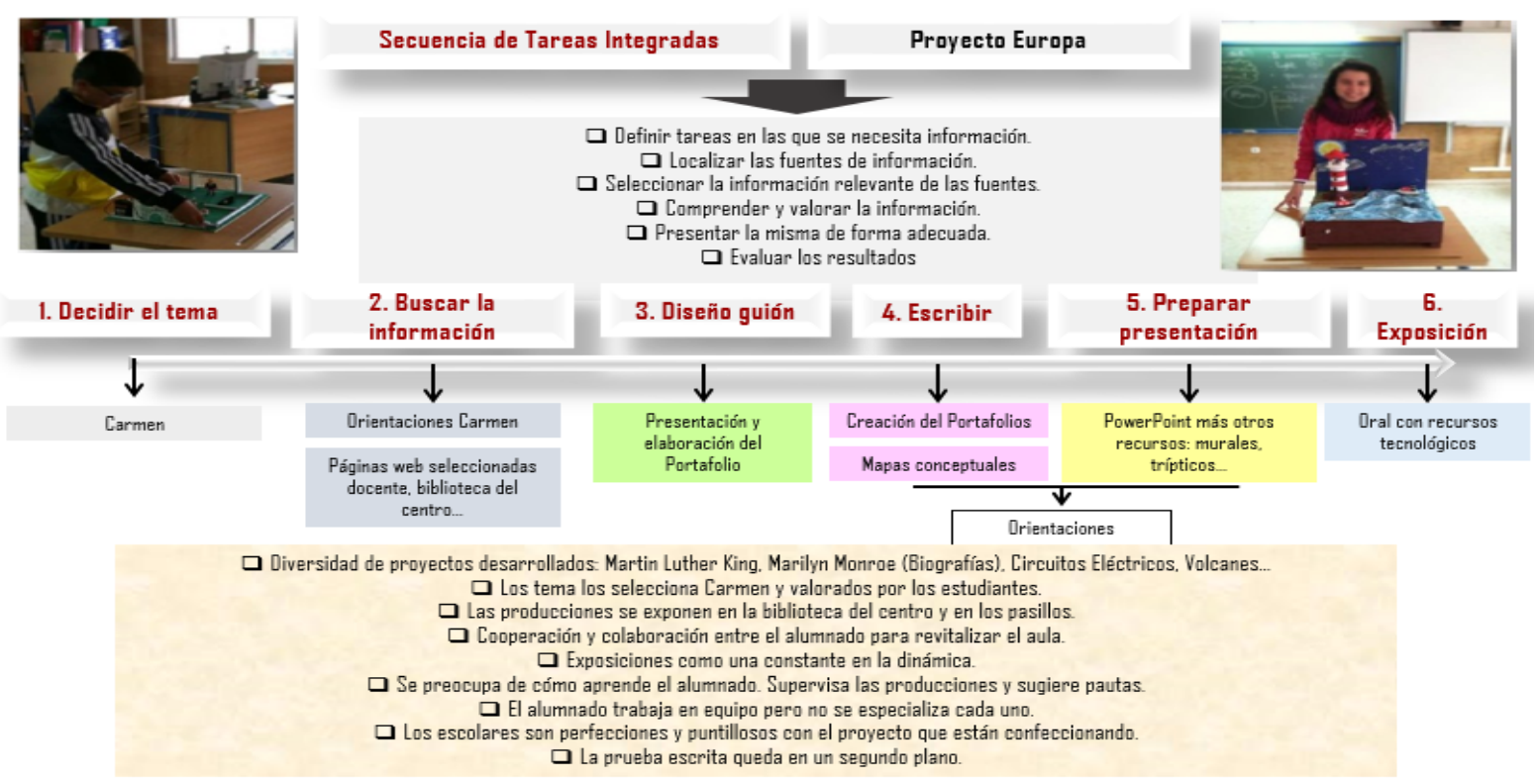

Figura 5. Desarrollo del "Proyecto Europa"

El clima que se respira es de un trabajo profundo y maduro por parte del alumnado que, poco a poco, se han ido iniciando en tareas integradas, garantizando, a su vez, un conocimiento coherente y organizado. No caben las actividades sencillas, pero sí aquellas que fomentan un pensamiento cognitivo superior. Se adapta a los cambios de la sociedad e introduce mecanismos para que el alumnado evolucione con ella.

El libro de texto está presente en ocasiones, pero las tareas integradas vislumbran un cambio progresivo en la práctica de Carmen, la estabilidad o la consolidación de los mismos en la acción educativa.

Los materiales que se emplean en el aula son diversos en función de las actividades de aprendizaje, destacando los materiales tecnológicos y los necesarios para la realización de maquetas que, en ocasiones, han realizado junto a otra serie de producciones.

La evaluación constituye un mecanismo para revisión de los aprendizajes, desde la observación y la atención. La prueba escrita queda en un segundo plano dando paso a las exposiciones orales y las observaciones diarias como el vehículo que guía el proceso evaluativo.

El aula se transforma con las actividades de aprendizaje. La clase se encuentra distribuida en forma de U. No cuenta con rincones pero hay espacios para que el alumnado exponga sus propias producciones.

Destacar que Carmen no es la tutora de sexto de primaria, por lo que el espacio se encuentra supeditado a las decisiones de las tutoras de sexto A y sexto B. No obstante, en la asignatura que ella imparte, distribuye el aula de manera flexible.

El alumnado es el que se encarga de "transformar" el aula. Al ser el trabajo en equipo el motor que da vida y movimiento al aula, la distribución de las mesas en grupo es un continuum en el grupo-clase. Señalar que para las exposiciones orales el espacio se amplía, dejando libertad de movimientos en el aula.

La estructura temporal se encuentra condicionada por la parcelación del conocimiento en disciplinas. Aún sigue imperando la rígida plantilla horaria, aunque con breves modificaciones parciales. A modo de ejemplo, Carmen introdujo una pequeña "metamorfosis" en dos asignaturas: 
Tutoría y Plástica. De forma consensuada con las tutoras, en este tiempo, Carmen mostraba al alumnado como realizar una presentación con el uso del PowerPoint y la exposición oral.

La organización espacial del aula crea y potencia un ambiente rico en aprendizajes colectivos, tomando como base la democracia y la capacidad de elegir de los estudiantes sobre la mejora de los espacios del aula.

Desde esta perspectiva de trabajo en la clase, se fomenta la cooperación y la utilización de estrategias de socialización ricas, las cuales permiten vincular las tareas con la realidad de los estudiantes y con la realidad social que circunda al centro educativo.

Carmen tiene en cuenta cómo trabajarán sus escolares, qué tipo de responsabilidad personal asume cada uno dentro del grupo, qué papel juega ella como docente dentro de la tarea. Las ideas se comparten y cada alumno es capaz de orientar al otro en la consecución de su propio aprendizaje. Los conflictos se solventan con diferentes dinámicas, tomando como base una adecuada educación emocional. Los grupos se conforman de forma heterogénea, depositando en los estudiantes un grado de confianza para organizar los mismos.

La participación familiar, en este caso, queda relegada al ámbito doméstico, con una razón clarificadora: Carmen al no ser tutora se encuentra condicionada en ese plano. Sin embargo, la docente diseña actividades que requieren la colaboración de las familias en el hogar.

Como limitaciones, la realidad familiar de muchos de los escolares supone una dificultad para la vida diaria en el centro -casos de absentismo localizado y relaciones difíciles entre familias-, la rígida plantilla horaria y la parcelación del conocimiento en disciplinas y el peso de las pruebas de diagnóstico al pasar de etapa educativa.

Para concluir, señalar algunos de los elementos que favorecen la actividad en el aula: la incorporación de las tareas integradas en la dinámica de enseñanza, el papel que la docente asume constituye el verdadero motor que hace progresar el grupo-clase y el trabajo en equipo como base de una enseñanza democrática y dialógica.

\section{Discusión y Conclusiones}

\section{Hacia nuevos planteamientos terminológicos del “aula” en la sociedad del siglo XXI}

El aula se percibe como un espacio donde la diversidad es su elemento más característico, en un entorno interactivo y sistémico. Es un lugar que forja interacciones, en un espacio social y socializador.

Las aulas de Julia y Carmen son diferentes, singulares y particulares. De ellas extraemos una serie de consideraciones básicas de las que aprender: un aula necesita de protagonistas, los propios escolares. Es un lugar de encuentro entre diferentes agentes de la comunidad educativa. Asimismo, el aula traspasa la frontera de sus paredes, abriéndose al mundo y al entorno.

La creatividad es otro de los puntos característicos. La clase es pura "esencia creativa" gracias al fluir de la imaginación de los escolares y a la aplicación de sus ideas en la resolución de problemas. Es un espacio comunicativo y que comunica.

Las aulas de ambas docentes se configuran como un álbum de vivencias y metáforas. El alumnado aprende a través de la colaboración, diálogo igualitario y negociación. Es un reflejo de múltiples influencias: familiares, sociales y culturales. 


\section{La estructura académica desde un enfoque integrado}

La enseñanza se construye en base al aprendizaje del alumnado y se enseña desde una perspectiva integrada, en el caso de Julia, y embrionaria en el caso de Carmen.

El conocimiento no se presenta cerrado, sino abierto, y se va construyendo en base a una enseñanza planificada y flexible. Se accede a él gracias a una diversidad de fuentes de información, integrando diferentes formas de conocimiento. Las ideas y los intereses del alumnado forman su piedra angular y la motivación se constituye como los cimientos del proceso.

El docente investiga y se actualiza, planifica los proyectos partiendo de los interrogantes del alumnado. Las temáticas son seleccionadas de acuerdo a la relevancia social, a la pertinencia curricular y sujeta a los intereses de los escolares.

Las actividades de aprendizaje promueven la expresión de interrogantes y problemas, impulsan el acceso a informaciones significativas. Se diseñan teniendo en cuenta diferentes niveles de complejidad intelectual. A su vez, permiten la incorporación de materiales diversos y diferentes.

La realidad denota un cambio importante: "hay algo más que el libro de texto", incluso en la etapa donde este material se encuentra más enraizado. Poco a poco los docentes van incluyendo "tentativas" que marcan pequeños pasos hacia la mejora de la práctica en el aula.

La evaluación acompaña el proceso de forma continua. Las revisiones de progreso son una constante, garantizando en el alumnado un compromiso hacia su propio aprendizaje, donde las altas expectativas están más que aseguradas.

Una evaluación que no se limita al examen, incorporando otras técnicas que evidencian el valor de las producciones del alumnado (observaciones, diario docente, trama conceptual, rúbricas de evaluación, carpetas de aprendizaje, exposiciones orales).

Asimismo, la reflexión sobre la práctica se arremete como propicia para la transformación de la labor profesional.

Se plantea una estructura académica donde la voz del alumnado es la clave, unido a enfoques integradores, a una evaluación formativa y a la apertura al medio.

\section{El espacio y el tiempo: promotores de un ambiente de aprendizaje diverso y adaptado a las características del alumnado}

El espacio facilita un determinado ambiente de aprendizaje, aunque las aulas de infantil y primaria continúan siendo desde el plano organizativo diferentes, pueden construir ambientes de aprendizaje diversos, estimulantes y activos.

Los espacios se flexibilizan, se amplían y se adecúan en función de las actividades de aprendizaje y de las distintas necesidades personales y escolares.

La riqueza de estímulos de la etapa de infantil envuelve al aula en un espacio organizativo particular, configurándose en torno a rincones, talleres, asamblea, así como otros espacios donde el juego libre fluye espontáneamente. Las producciones de los escolares marcan la personalidad y la historia del aula. necesario.

El aula de Carmen posibilita la configuración del espacio en grupos de trabajo cuando es

Se rompe con la concepción tradicional del tiempo, en la medida de lo posible, aunque siguen existiendo barreras al respecto, principalmente en la Educación Primaria.

Existen espacios temporales para interrogarse, plantear hipótesis, sugerir actividades, para buscar, obtener datos e informaciones. Tiempo para elaborar conclusiones, reflexionar, expresar e intervenir en la realidad. 


\section{La construcción de un espacio favorecedor de interacciones dialógicas, democráticas y comunicativas entre el alumnado}

Queda latente que, en ambas experiencias, el aula se convierte en un espacio favorecedor de interacciones dialógicas, democráticas y comunicativas entre el alumnado.

La asamblea en algunas experiencias y el diálogo en otras, disfruta de unos espacios y tiempos determinados. En ella se suelen tratar problemas y cuestiones que afectan a la vida del aula, así como, experiencias, sentimientos, etc.

Las relaciones personales que se establecen en el aula se basan en el respeto, en la aceptación de los demás, en la cooperación, en la ayuda y en la equidad. En los casos descritos se pone de manifiesto que las relaciones interpersonales son bastante enriquecedoras.

Se concibe el aula como un espacio en el que los escolares tratan de ayudarse los unos a los otros, siendo el docente y otros agentes que intervienen, los principales apoyos para el alumnado.

La clase se convierte en una pequeña comunidad con unos principios claros: cohesión social, justicia, equidad, diálogo. Resulta necesario el debate y propuestas que impliquen la ayuda entre los escolares. En ambos casos, se recurre al trabajo en pequeños grupos donde el conocimiento se construye de forma compartida, gracias a la labor del equipo, sin dejar de lado la perspectiva individual.

\section{La participación comunitaria como ejes de una dinámica que evoluciona} colaborar.

Se aprende de los otros sí, pero sólo si les damos la oportunidad de participar y de

En el aula de Julia comprobamos que la participación comunitaria es cuidada como cualquier elemento de la planificación didáctica. Los "otros agentes" asumen el papel de impulsar la práctica de Julia, son su más fiel apoyo. Carmen se encuentra más limitada, al no ser tutora, pero diseña actividades que necesita la ayuda de las familias.

La gramática de la escuela evita el formalismo de su ajuar para incidir en lo colectivo desde las puertas de la participación.

La acción educativa ha de impulsar la creación de redes de participación comunitaria. Escuchar las voces de los otros, plantear y diseñar acciones conjuntas con el fiel propósito de la mejora de la práctica en el aula.

Comunidad y Escuela, un binomio particular con un mismo propósito.

Las experiencias innovadoras se impulsan gracias a equipos comprometidos con diferentes puntos de vista y singularidades, pero con propósitos compartidos: "Caminando paso a paso, lentamente, evitando un ritmo frenético, a tanto por hora, también llegamos”.

Numerosas prácticas educativas se han visto abocadas al fracaso por el abandono progresivo de sus miembros. Las disputas y los desencuentros siempre van a estar presentes, sin embargo, la fortaleza de un equipo se gana con el diálogo conjunto.

\section{No importa cómo nos encontremos sino hacia dónde vamos}

El trabajo compartido impulsa la actividad en el aula. Julia a veces se siente pesimista ante las múltiples vicisitudes que tienen como punto de mira a los centros escolares, pero sigue primando su esencia docente.

Julia y Carmen buscan y encuentran alternativas. No se dejan llevar por la oleada de pesimismo, aunque a veces "las roza". En cambio, reflexionan y planifican medidas para favorecer 
la construcción de aprendizajes significativos, desde el diálogo, el debate, las actividades de aprendizaje, entre otras.

Lo que está claro en ellas, es que ponen de manifiesto cómo las innovaciones y el grado de éxito de estas iniciativas son fruto de un esfuerzo progresivo, en evolución permanente y no siempre de fácil mantenimiento. En cada caso, se puede observar como cada docente despliega su capacidad para mejorar la cotidianidad de sus aulas. Teniendo en cuenta que existen momentos difíciles y obstáculos que muchas veces dificultan que prácticas como éstas se lleven a cabo.

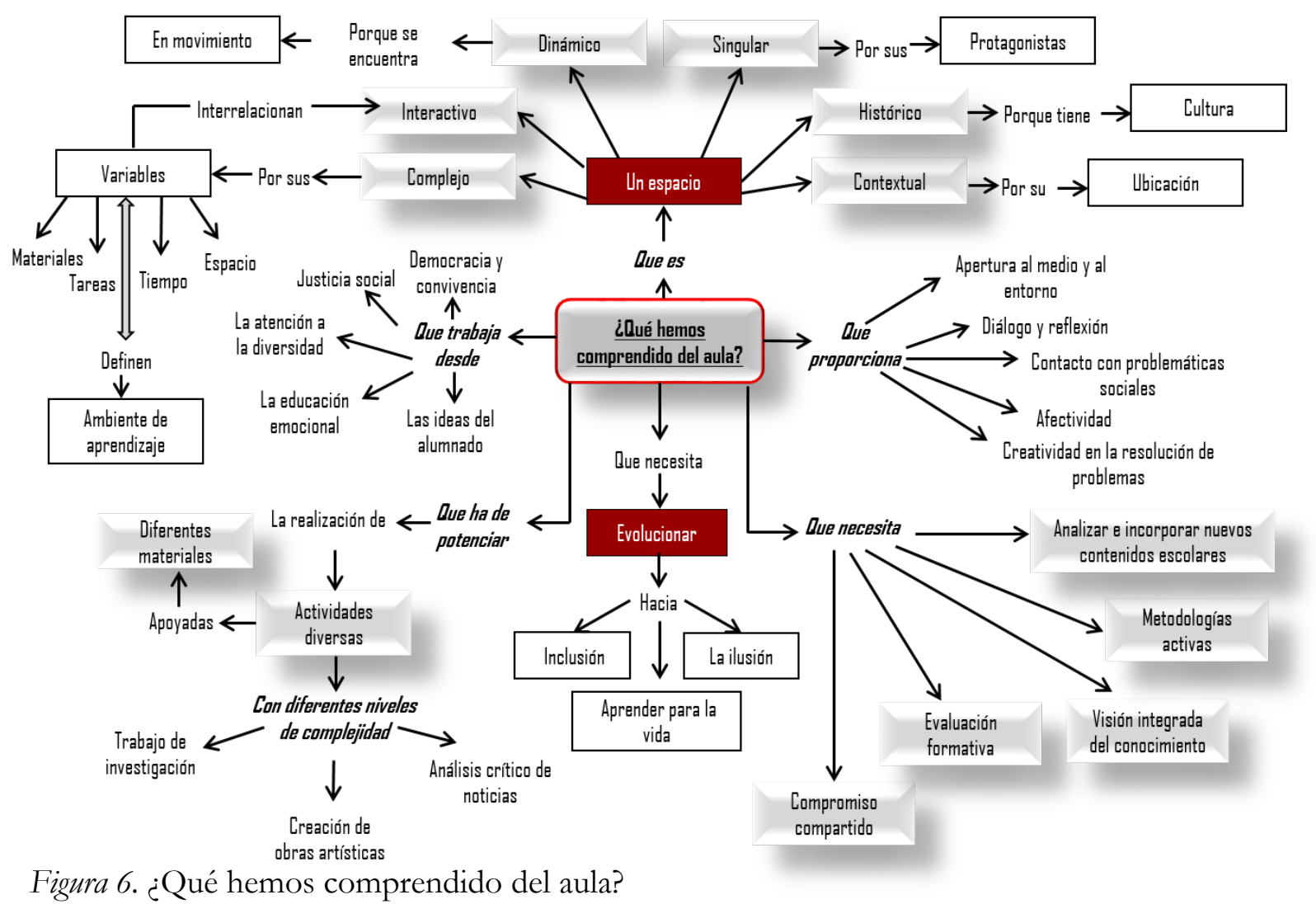

Figura 6. ¿Qué hemos comprendido del aula?

\section{Referencias}

Argos, J. (2008). Procesos de subjetivación y construcción de la ciudadanía desde el contexto escolar. En J. Marrero y J. Argos (Coords.), Educación, convivencia y ciudadanía en la cultura global (pp. 47-71). Bilbao: Wolters Kluwer.

Belavi, G. y Murillo, F.J. (2016). Educación, Democracia y Justicia Social. Revista Internacional de Educación para la Justicia Social (RIEJS), 5 (1), 13-34.

Bolívar, A. (2012). Políticas actuales de mejora y liderazgo educativo. Archidona: Aljibe. Carnie, F. (2003). Alternative Approaches to Education. Londres: Routledge Falmer.

Cook-Sather, A. (2007). Resisting the impositional potential of student voice work: Lessons for liberatory educational research from poststructuralist feminist critiques of critical pedagogy. Discourse, 28 (3), 389-403.

Escudero, J.M. (1999). Diseño, desarrollo e Innovación del currículum. Madrid: Síntesis. 
Fielding, M. (2011). La voz del alumnado y la inclusión educativa: una aproximación democrática radical para el aprendizaje intergeneracional. Revista Interuniversitaria de Formación del Profesorado, 70 (25,1), 31-61.

Fullan, M. (2002). Los nuevos significados del cambio en la educación. Barcelona: Octaedro.

Gimeno, J. (2005). La educación que aún es posible. Ensayos acerca de la cultura para la educación. Madrid: Morata.

Giné, C., Durán, D., Font, J. y Miquel, E. (Eds.) (2009). La educación inclusiva. De la exclusión a la plena participación de todo el alumnado. Barcelona: Horsori Editorial.

Giroux, H.A. (2003). Pedagogía y política de la esperanza. Buenos Aires: Amorrortu editores.

Goetz, J.P. y Lecompte, M.D. (1988). Etnografía y diseño cualitativo en investigación educativa. Madrid: Morata.

Gutiérrez, R. (2006). Los estudios de casos una opción metodológica para investigar la educación artística. En R. Martín (Coord.), Investigación en educación artística. Granada: Universidades de Granada y de Sevilla.

Harris, A. y Jones, M. (2010). Professional learning communities and system improvement. Improving Schools, 13 (2), 172-181.

Murillo, F.J. y Krichesky, G.J. (2015). Mejora de la Escuela: Medio siglo de lecciones aprendidas. REICE. Revista Iberoamericana sobre Calidad, Eficacia y Cambio en Educación, 13 (1), 69-102.

Parrilla, A., Muñoz, M.A. y Sierra, S. (2013). Proyectos Educativos con vocación comunitaria. Revista de Investigación en Educación, 11 (3), 15-31.

Pérez, E.M. (2004). La influencia de variables familiares, personales y escolares en los resultados de los alumnos. (Tesis Doctoral). Madrid: Servicio de Publicaciones de la Universidad Complutense de Madrid.

Pozuelos, F.J. (2007). Trabajo por proyectos en el aula: descripción, investigación y experiencias. Morón de la Frontera (Sevilla): Cooperación Educativa.

Pozuelos, F.J., González, A. y Travé, G. (2008). Investigando la alimentación bumana. Proyecto Curricular Investigando Nuestro Mundo (6-12). Sevilla: Díada Editora.

Pozuelos, F.J., Romero, D., García, F.J. y Morcillo, V. (2010). No basta con soñar otra escuela, hay que hacerla. Relatos de experiencias y un caso. Investigación en la Escuela, 70, 5-20.

Subirats, J. (2010). Evaluar desde el reconocimiento de la diversidad. La evaluación de políticas públicas de un sistema altamente descentralizado. El caso de España. Análisis local, 88, 7-14.

Susinos, T. y Ceballos, N. (2012). Voz del alumnado y presencia participativa en la vida escolar. Apuntes para una cartografía de la voz del alumnado en la mejora educativa. Revista de Educación, 319, 24-44.

Thurler, M. (2004). Innovar en el seno de la institución escolar. Barcelona: Graó.

Trillo Alonso, F. (1994). El profesorado y el desarrollo curricular: tres estilos de hacer la escuela. Cuadernos de pedagogía, 228, 70-74.

\section{Información sobre la autora}

Autora: Virginia Morcillo Loro

Institución: Universidad de Huelva

Email: virmorcilloloro@gmail.com 


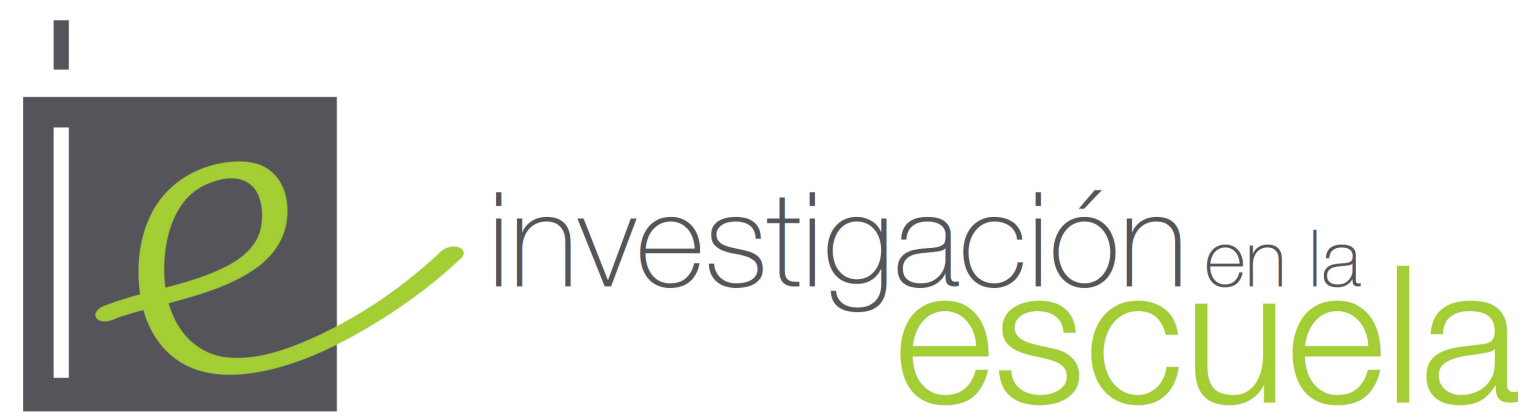

Revista académica evaluada por pares y de acceso abierto

Número 92

07 de septiembre de 2017

ISSN 2443-9991

\section{(c)}

SOMRERIGHISRESERVEDLOS/as lectores/as pueden copiar, mostrar, y distribuir este artículo, siempre y cuando se de crédito y atribución al autor/es y a Investigación en la Escuela, se distribuya con propósitos no-comerciales, no se altere o transforme el trabajo original. Más detalles de la licencia de CreativeCommons se encuentran en http://creativecommons.org/licenses/by-nc-sa/3.0 Cualquier otro uso debe ser aprobado en conjunto por el autor/es, o Investigación en la Escuela.

ư investigacion-en-la-escuela

Contribuya con comentarios y sugerencias en la web de la revista. Por errores y sugerencias contacteasecretaria@investigacionenlaescuela.es 


\section{Investigación en la escuela}

Consejo de dirección: Ana Rivero García (Universidad de Sevilla), Nicolás de Alba Fernández(Universidad de Sevilla), Pedro Cañal de León (Universidad de Sevilla), Francisco F. García Pérez (Universidad de Sevilla), Gabriel Travé González, (Universidad de Huelva), Francisco F. Pozuelos Estrada (Universidad de Huelva)

Dirección: Ana Rivero García y Nicolás de Alba Fernández

Secretaría de edición: Elisa Navarro Medina

\section{Consejo editorial}

José Félix Angulo Rasco. Universidad de Cádiz Rosa Ma Ávila Ruiz. Universidad de Sevilla Pilar AzcárateGoded. Universidad de Cádiz Juan Bautista Martínez Rodríguez. Universidad de Granada

Nieves Blanco García. Universidad de Málaga Fernando Barragán Medero. Universidad de La Laguna José Carrillo Yáñez. Universidad de Huelva José Contreras Domingo. Universidad de Barcelona. Luis C. Contreras González. Universidad de Huelva Ana $\mathbf{M}^{\mathbf{a}}$ Criado García-Legaz. Universidad de Sevilla Rosario Cubero Pérez. Universidad de Sevilla José $\mathbf{M}^{\mathbf{a}}$ Cuenca López. Universidad de Huelva Jesús Estepa Giménez. Universidad de Huelva Rafael Feito Alonso. Universidad Complutense (Madrid)

Francisco José García Gallardo. Universidad de Huelva

Soledad García Gómez. Universidad de Sevilla J. Eduardo García Díaz. Universidad de Sevilla
Fernando Hernández Hernández. Universidad de Barcelona

Salvador Llinares Ciscar. Universidad de Alicante Alfonso Luque Lozano. Universidad de Sevilla Rosa Martín del Pozo. Universidad Complutense (Madrid)

José Martín Toscano. IES Fernando Herrera (Sevilla) Jaume Martínez Bonafé. Universidad de Valencia F. Javier Merchán Iglesias. Universidad de Sevilla Emilia Moreno Sánchez. Universidad de Huelva.Rosario Ortega Ruiz. Universidad de Córdoba

Antonio de Pro Bueno. Universidad de Murcia Fco. de Paula Rodríguez Miranda. Universidad de Huelva

Pedro Sáenz-López Buñuel. Universidad de Huelva Antoni Santisteban Fernández. Universidad Autónoma (Barcelona) Emilio Solís Ramírez. Catedrático de IES. $\mathbf{M}^{\mathbf{a}}$ Victoria Sánchez García. Universidad de Sevilla. Magdalena Suárez Ortega. Universidad de Sevilla

\section{Consejo asesor}

Manuel Área Moreira. Universidad de La Laguna

Jaume Carbonell. Director Cuadernos de Pedagogía. Barcelona

César Coll. Universidad de Barcelona

Christopher Day. Universidad de Nothingham. U.K.

Juan Delval. Universidad Nacional de Educación a Distancia

John Elliott. Universidad de East Anglia. Norwich. U.K.

José Gimeno Sacristán. Universidad de Valencia

André Giordan. Universidad de Paris VII y Ginebra

Francisco Imbernón. Universidad de Barcelona

Ángel Pérez Gómez. Universidad de Málaga

Rafael Porlán Ariza. Universidad de Sevilla

Francesco Tonucci. Instituto de Pedagogía del C.N.R. Roma

Jurjo Torres Santomé. Universidad de A Coruña 
